\title{
Quantum Groups and Generalized Statistics in Integrable Models
}

\author{
F. A. Smirnov \\ Leningrad Branch of Steklov Mathematical Institute, \\ Fontanka 27, SU-191011 Leningrad, USSR
}

\begin{abstract}
The paper deals with the integrable massive models of quantum field theory. It is shown that generalized statistics of physical particles is closely connected with the invariance under quantum groups. This invariance provides the possibility to construct quasi-local operators (parafermions) possessing generalized statistics which interpolates the physical particles. For the particular examples of SG, RSG models and scaling 3-state Potts model the parafermions are described completely (all their matrix elements in the space of states are presented).
\end{abstract}

\section{Introduction}

In two-dimensional space-time the quantum fields can satisfy the commutation relations which generalize the usual bosonic and fermionic ones [1, 2]. The most general form of these relations is

$$
\begin{gathered}
O_{i}(x) O_{j}(y)=O_{\ell}(y) O_{k}(x) R_{i j}^{k \ell}, \\
x<y
\end{gathered}
$$

where $x, y$ are space coordinates. The matrix $R$ should be a constant solution of the Yang-Baxter equation:

$$
R_{12} R_{13} R_{23}=R_{23} R_{13} R_{12} .
$$

Here we used usual tensor notations $\left(R_{i j}\right.$ acts nontrivially in the tensor product of $i^{\text {th }}$ and $j^{\text {th }}$ spaces). Even diagonal matrices $R$ are of interest in this context; they correspond, for example, to kinks in the Gross-Neveu Model [2] or parafermions in $Z_{N}$ models [3]. The commutation relations (0.1) with nontrivial $R$-matrices appeared originally in the framework of conformal field theory [4-6].

In the present paper we show how to realize the relations $(0.1)$ for massive completely integrable models and explain the connection between generalized statistics and symmetries associated with quantum groups. The $S$-matrix in the completely integrable model is factorizable. The two-particle $S$-matrix should 
satisfy the equation of factorization [7] (Yang-Baxter equation $[8,9]$ ):

$$
S_{12}\left(\beta_{1}-\beta_{2}\right) S_{13}\left(\beta_{1}-\beta_{3}\right) S_{23}\left(\beta_{2}-\beta_{3}\right)=S_{23}\left(\beta_{2}-\beta_{3}\right) S_{13}\left(\beta_{1}-\beta_{3}\right) S_{12}\left(\beta_{1}-\beta_{2}\right),
$$

where $\beta_{1}, \beta_{2}, \beta_{3}$ are the rapidities of particles. The matrix $S(\beta)$ is analytical function of $\beta$. For many interesting models the $S$-matrix possesses nontrivial asymptotics for $\beta \rightarrow \pm \infty$ :

$$
S_{12}(\beta) \underset{\beta \rightarrow \infty}{\longrightarrow} R_{12}, \quad S_{12}(\beta) \underset{\beta \rightarrow-\infty}{\longrightarrow}\left(R_{21}\right)^{-1},
$$

where $R_{21}=P R_{12} P, P$ is the operator of permutation, $R$ is a constant solution of Yang-Baxter equations. This behaviour contradicts a certain physical intuition which requires that the amplitude of elastic scattering should tend to 1 when the corresponding $S$-variable tends to infinity. This problem was discussed in the papers $[1,2]$ where it was proposed to introduce instead of the particles with the $S$-matrix $S(\beta)$ which are considered as auxiliary ones, the physical particles possessing generalized statistics,

$$
a_{\text {out } i}^{*}\left(\beta_{1}\right) a_{\text {out } j}^{*}\left(\beta_{2}\right)=a_{\text {out } \ell}^{*}\left(\beta_{2}\right) a_{\text {out } k}^{*}\left(\beta_{1}\right) R_{i j}^{k \ell}, \quad \beta_{1}<\beta_{2} .
$$

The $S$-matrix of the physical particles is not analytical:

$$
\tilde{S}_{12}(\beta)=S_{12}(\beta)\left(R_{12}^{-1} \theta(\beta)+R_{21} \theta(-\beta)\right),
$$

but it satisfies the condition

$$
\tilde{S}_{12}(\beta) \rightarrow I, \quad \beta \rightarrow \pm \infty .
$$

It is very easy to pass from the base of auxiliary particles to the base of physical ones. Technically the auxiliary particles are more suitable. However the principal statement is that if $S$-matrices possess nontrivial asymptotics we actually deal with physical particles satisfying generalized statistics.

Evidently Eqs. (0.1) and (0.3) are closely connected. The interpolating fields of the particles satisfying $(0.3)$ should satisfy $(0.1)$. Here a crucial difference with usual bosonic or fermionic statistics appears: the fields satisfying (0.1) cannot be expressed via the Fourier transform through the creation-annihilation operators satisfying $(0.2)$. That is why the theories for which the relations $(0.1),(0.3)$ hold should be nonlinear, i.e. they necessarily contain nontrivial interaction. In the present paper it will be shown that the relations $(0.1),(0.3)$ can be realized in integrable models. Evidently, integrable models are the simplest ones for which it can be done, so, they play the same role for the relations $(0.1),(0.2)$ as the free fields play for usual bosonic and fermionic relations.

From another point of view the relations $(0.3)$ should be connected with invariance of the theory under the action of quantum group associated with the matrix $R$ [10-12]. An example of the phenomenon is considered in the paper [13]. We shall suppose that the models under consideration do possess quantum group invariance. For particular models it can be checked using the explicit formulas for $S$-matrices and form factors of local operators [14-16].

We shall show that for the models with $S$-matrices possessing asymptotics $(0.2)$ it is possible to construct the parafermionic operators $\psi, \bar{\psi}$ (to construct the 
means to present all their matrix elements in the space of states) which satisfy the following commutation relations and asymptotic conditions:

$$
\begin{aligned}
& \psi_{i}\left(x_{0}, x_{1}\right) \psi_{j}\left(x_{0}, x_{1}^{\prime}\right)=\psi_{\ell}\left(x_{0}, x_{1}^{\prime}\right) \psi_{k}\left(x_{0}, x_{1}\right) R_{i j}^{k \ell}, \\
& x_{1}<x_{1}^{\prime} \text {; } \\
& \bar{\psi}_{i}\left(x_{0}, x_{1}\right) \bar{\psi}_{j}\left(x_{0}, x_{1}^{\prime}\right)=\bar{\psi}_{\ell}\left(x_{0}, x_{1}^{\prime}\right) \bar{\psi}_{k}\left(x_{0}, x_{1}\right) R_{i j}^{k \ell} \text {, } \\
& x_{1}>x_{1}^{\prime} \text {; } \\
& \psi_{i}\left(x_{0}, x_{1}\right) \bar{\psi}_{j}\left(x_{0}, x_{1}^{\prime}\right)=\bar{\psi}_{\ell}\left(x_{0}, x_{1}^{\prime}\right) \psi_{k}\left(x_{0}, x_{1}\right) R_{i j}^{k \ell} \text {, } \\
& \forall x_{1}, x_{1}^{\prime} ;
\end{aligned}
$$

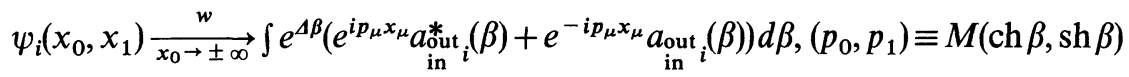

$$
\begin{aligned}
& \bar{\psi}_{i}\left(x_{0}, x_{1}\right) \underset{x_{0} \rightarrow \pm \infty}{\stackrel{w}{\longrightarrow}} \int e^{-\Delta \beta}\left(e^{i p_{\mu} x_{\mu}} a_{\text {in }}^{*}{ }_{i}^{*}(\beta)+e^{-i p_{\mu} x_{\mu}} a_{\text {in }}{ }_{\text {out }}(\beta)\right) d \beta,
\end{aligned}
$$

where $\Delta$ is "spin" of the parafermion. In the scaling limit $\psi, \bar{\psi}$ give parafermionic currents in CFT [17].

The paper is organized as follows. In Sect. 1 we give an introduction to quantum groups. In Sect. 2 we summarize necessary facts about integrable models, mainly about the construction of local operators in terms of physical particles. In Sect. 3 we consider the quantum group invariance and present the general construction of $\psi, \bar{\psi}$. Sections 4 and 5 are devoted to the consideration of particular examples. In Sect. 4 we construct the operators $\psi, \bar{\psi}$ in SG model which is invariant under $S L(2)_{q}$, and in RSG models which are reductions of SG $[13,18]$. The models RSG coincide $[13,18]$ with the perturbation of minimal models of CFT by the operator $\phi_{13}$ [19-21]. In Sect. 5 we consider the scaling 3-state Potts model which is the perturbation of CFT $M_{5}$ by the operator $\phi_{21}$ [21]. In Sect. 6 we give some additional remarks concerning the subject of the paper.

\section{Quantum Groups}

Quantum groups defined in the papers [10-12] give examples of noncommutative and noncocommutative Hopf algebras. The most important property of Hopf algebras is the possibility to construct the tensor products of their representations. This property allows to use quantum groups as generalizations of isotopic groups in QFT (see for example the paper [13] where the invariance of SG under $S L(2)_{q}$ is established). In this section we give an introduction to quantum groups.

The simplest example of a Hopf algebra is the algebra of functions on Lie group. Consider for example the group $S L(2)$. Let $A$ be the algebra of functions on $G: f \in A, f: G \rightarrow \mathbb{C}$. The algebra $A$ has a natural set of generators $g_{i}^{j}(i, j=1,2)$ which are the functions whose values on arbitrary $h \in G$ give matrix elements of $h$ in twodimensional representation:

$$
g_{i}^{j}(h)=h_{i}^{j} .
$$

The multiplication in $A$ is the usual point-wise multiplication of functions. There is also an operation of comultiplication $\Delta: \Delta: A \rightarrow A \otimes A, \Delta$ is homomorphism. On 
generators comultiplication is defined as follows:

$$
\Delta\left(g_{i}^{j}\right)=\sum_{k} g_{k}^{j} \otimes g_{i}^{k} .
$$

The counit is the homomorphism $A \rightarrow \mathbb{C}$ defined on generators as

$$
\varepsilon\left(g_{i}^{j}\right)=\delta_{i}^{j} .
$$

An important requirement is in existence of the antipode $s: A \rightarrow A$ which is an antiautomorphism of algebras in the particular case under consideration

$$
s(g)=\sigma^{2} g^{t} \sigma^{2}
$$

where $g_{i}^{j}$ are combined into a $2 \times 2$ matrix $g$. This formula gives an inverse element to $g$ considered as a matrix from $S L(2)$. One has

$$
s(g) g=g s(g)=I \text {. }
$$

We can reformulate all the theory of Lie groups in these terms. This way seems to be exotic but it appears to be very useful for generalization. Notice in particular that the representations of the group $S L(2)$ is in one-to-one correspondence with corepresentations of the algebra $A$. Corepresentation is matrix $t$ whose matrix elements belong to $A$, and for which the formula of comultiplication is

$$
\Delta(t)=t \dot{\otimes} t,
$$

where - means matrix multiplication. The matrix $g$ itself is the simplest example of corepresentation (the two-dimensional one).

Let us now consider the noncommutative generalization of this construction. Suppose we have noncommutative algebra $A$ whose generators can be combined into the matrix $g$ which satisfy the following properties:

1. The algebra $A$ is a noncommutative algebra with unit 1 . The $(N \times N)$ matrix of generators $g$ satisfies the relation

$$
R_{12} g_{1} g_{2}=g_{2} g_{1} R_{12},
$$

where the right-hand side and left-hand side are operators in the tensor square of $\mathbb{C}^{N}, g_{1}=g \otimes I, g_{2}=I \otimes g$. The matrix $R_{12}$ is invertible $N^{2} \times N^{2} \mathbb{C}$-number matrix acting in $\mathbb{C}^{N} \otimes \mathbb{C}^{N}$.

2. There is the homomorphism $\Delta: A \rightarrow A \otimes A$ which is defined on generators as

$$
\Delta g=g \dot{\otimes} g \text {. }
$$

3. The counit $\varepsilon$ is the homomorphism $A \rightarrow \mathbb{C}$, defined on generators as follows:

$$
\varepsilon(g)=I \text {. }
$$

4. There is an antiautomorphism of $A$ which is called antipode $s$. For generators one has

$$
g s(g)=s(g) g=I .
$$

Suppose we are provided with the Hopf algebra which satisfies these requirements, then we say that we deal with the quantum group. However, one should not 
identify the quantum group with algebra $A$. The quantum group itself is an object which cannot be described in inner terms. The algebra $A$ is "the algebra of functions on the quantum group" [12]. The representation of the quantum group is the corepresentation of $A$, i.e. matrix $t$ with elements from $A$ for which the comultiplication is of form

$$
\Delta t=t \dot{\otimes} t
$$

In what follows two special representations of $A$ will be important. The associativity and independence of cubic monomes requires that $R$ satisfies the Yang-Baxter equation, $\quad R_{12} R_{13} R_{23}=R_{23} R_{13} R_{12}$.

Define the homomorphism of algebras $\varrho^{+}: A \rightarrow \operatorname{Mat}_{N}(\mathbb{C})$ :

$$
\left(\varrho^{+}\left(g_{i}^{j}\right)\right)_{k}^{\ell}=R_{i k}^{j \ell} .
$$

The formula (1.3) means that we divide $R_{12}$ into blocks with respect to the first space which correspond to $g_{j}^{i}$ under the homomorphism $\varrho^{+}$. We shall write (1.3) as follows:

$$
\varrho_{2}^{+}\left(g_{1}\right)=R_{12} \text {. }
$$

From (1.1) and (1.2) it follows that $\varrho^{+}$is really a homomorphism of algebras. In the relation (1.1) $R$ is defined up to a normalization. In all the particular cases the normalization can be fixed in order that $\varrho^{+}(g)$ satisfies requirement 4 . Then $\varrho^{+}$is a homomorphism of Hopf algebras. Similarly we define the homomorphism $\varrho^{-}(\mathrm{g})$ :

$$
\varrho_{2}^{-}\left(g_{1}\right)=\left(R_{21}\right)^{-1} \text {. }
$$

We would like to emphasize that $\varrho^{ \pm}$are representations of Hopf algebra $A$, but they are not representations of the quantum group which are corepresentations of $A$.

Let us consider particular examples which will be used in this paper.

Example 1. The group $Z_{3}$. This is a trivial example which demonstrates the general character of the above construction. The matrix of generators $g$ is $1 \times 1$ matrix, i.e. we deal with the algebra with one generator.Evidently $R$ can be chosen as an arbitrary constant. We prefer $R=\exp (2 \pi i / 3)$. The antipode $s(g)$ is given by

$$
s(g)=g^{2} \text {. }
$$

The representations $\varrho^{ \pm}$are

$$
\varrho^{+}(g)=\exp \left(\frac{2 \pi i}{3}\right), \quad \varrho^{-}(g)=\exp \left(-\frac{2 \pi i}{3}\right) .
$$

Example 2. The quantum group $S L(2)_{q}$. The generators $g_{i}^{j}$ are combined into a $2 \times 2$ matrix $g$. The matrix $R$ is equal to

$$
R_{12}(q)=q^{-1 / 4}\left(\begin{array}{cccc}
q^{1 / 2} & & & \\
& 1 & & \\
q^{1 / 2}-q^{-1 / 2} & 1 & \\
& & & q^{1 / 2}
\end{array}\right)
$$


$q$ is the parameter. The antipode is given by

$$
s(g)=\alpha g^{t} \alpha,
$$

where

$$
\alpha=\left(\begin{array}{ll}
q^{-1 / 4} \\
-q^{1 / 4}
\end{array} .\right.
$$

It is easy to check that $\varrho^{ \pm}$do realize the representations of $A$. The representation theory of $S L(2)_{q}$ for generic $q\left(q^{r} \neq 1\right)$ is absolutely similar to that of $S L(2)$. For $q^{r}=1$ certain modifications arise [13]; we shall consider them partly in Sect. 4.

\section{Local Operators in Massive Integrable Models}

Consider some massive integrable model. For the sake of simplicity we suppose that the spectrum of the model contains only one particle which possesses isotopic degrees of freedom. Another assumption is that this particle is not a bound state of two particles, i.e. the theory does not contain a " $\varphi^{3}$-type interaction." So, we concentrate our attention on the models with one particle and no bound states. The generalization to the theory with bound states will be considered in Sect. 5 for the particular example of the Scaling Potts model.

The two-particle $S$-matrix can be considered as an operator acting in the tensor product of two isotopic spaces $S_{12}(\beta)$. The matrix $S(\beta)$ is analytical, it satisfies the requirements:

$$
\begin{gathered}
S_{12}\left(\beta_{1}-\beta_{2}\right) S_{13}\left(\beta_{1}-\beta_{3}\right) S_{23}\left(\beta_{2}-\beta_{3}\right)=S_{23}\left(\beta_{2}-\beta_{3}\right) S_{13}\left(\beta_{1}-\beta_{3}\right) S_{12}\left(\beta_{1}-\beta_{2}\right), \\
S_{12}(\beta) S_{21}(-\beta)=I \\
S_{12}(\pi i-\beta)=c_{2} S_{21}^{t_{2}}(\beta) c_{2}
\end{gathered}
$$

where $S_{21}=P_{12} S_{12} P_{12}, P_{12}$ permutes the isotopic spaces, $c_{2}$ is the matrix of the charge conjugation $c$ acting in the second space. We require that $c^{2}=I$. Let us notice that we do not require here the unitarity of the $S$-matrix: the equation $S_{12}(\beta) S_{21}(-\beta)=I$ does not necessarily correspond to unitarity. Generally, it is useful to delay the discussion of Hermitian conjugation and related problems. Now we present the formal construction considering the space of states and conjugated space as dual linear spaces.

Following the papers [14-16] we introduce the set of vectors in the space of states and the dual space

$$
\left|\beta_{n} \ldots \beta_{1}\right\rangle,\left\langle\beta_{1} \ldots \beta_{n}\right| \text {. }
$$

If the theory possesses isotopic degrees of freedom these vectors lie in tensor product of isotopic spaces. The vectors (2.2) satisfy the equations

$$
\begin{aligned}
& \left|\beta_{n}, \ldots \beta_{i+1}, \beta_{i} \ldots \beta_{1}\right\rangle S_{i i+1}\left(\beta_{i}-\beta_{i+1}\right)=\left|\beta_{n}, \ldots \beta_{i}, \beta_{i+1} \ldots \beta_{1}\right\rangle P_{i+1}, \\
& S_{i+1 i}\left(\beta_{i+1}-\beta_{i}\right)\left\langle\beta_{1}, \ldots \beta_{i}, \beta_{i+1} \ldots \beta_{n}\right|=P_{i+1}\left\langle\beta_{1}, \ldots \beta_{i+1}, \beta_{i} \ldots \beta_{n}\right| .
\end{aligned}
$$


For $\beta_{n}>\ldots>\beta_{1}\left(\beta_{n}<\ldots<\beta_{1}\right)$ the vectors (2.2) coincide with "in" ("out") states. The following pairings take place:

$$
\begin{gathered}
\left\langle\alpha_{1} \ldots \alpha_{m} \mid \beta_{n} \ldots \beta_{1}\right\rangle=\delta_{m n} \prod \delta\left(\alpha_{i}-\beta_{i}\right) I, \\
\alpha_{m}>\ldots>\alpha_{1}, \beta_{n}>\ldots>\beta_{1},
\end{gathered}
$$

where $I$ is the tensor which has the following index form: $I \sim \delta_{i_{1}}^{i_{1}^{\prime}} \ldots \delta_{i_{n}}^{i_{n}^{\prime}}\left(i_{1}^{\prime} \ldots i_{n}^{\prime}\right.$ and $i, \ldots i_{n}$ are isotopic indices corresponding to the particles $\alpha_{1} \ldots \alpha_{n}$ and $\beta, \ldots \beta_{n}$ ).

Consider the matrix elements of some local operator $O(0,0)$ :

$$
\left\langle\alpha_{1} \ldots \alpha_{m}|O(0,0)| \beta_{n} \ldots \beta_{1}\right\rangle=f\left(\alpha_{m} \ldots \alpha_{1} \mid \beta_{1} \ldots \beta_{n}\right)+\ldots,
$$

where dots mean the terms containing $\delta$-functions $\delta\left(\alpha_{i}-\beta_{j}\right)$ [15]. These matrix elements can be expressed in terms of formfactors

$$
f\left(\beta_{1} \ldots \beta_{n}\right) \equiv\left\langle 0|O(0,0)| \beta_{n} \ldots \beta_{1}\right\rangle
$$

according to the formula

$$
f\left(\alpha_{m} \ldots \alpha_{1} \mid \beta_{1} \ldots \beta_{n}\right)=c_{1} \ldots c_{m} f^{t_{1} \ldots t_{m}}\left(\alpha_{m}-\pi i, \ldots \alpha_{1}-\pi i, \beta_{1} \ldots \beta_{n}\right),
$$

where $c_{i}$ is the matrix of charge conjugation acting in the isotopic space associated with the particle $\alpha_{i}, t_{i}$ means transposing with respect to this space.

According to the results of the papers [15] the locality of the operator $O$ is equivalent to the following properties of formfactors:

1. $f\left(\beta_{1} \ldots \beta_{i}, \beta_{i+1} \ldots \beta_{n}\right) S_{i i+1}\left(\beta_{i}-\beta_{i+1}\right)=f\left(\beta_{1} \ldots \beta_{i+1}, \beta_{i} \ldots \beta_{n}\right) P_{i i+1}$.

2. $f\left(\beta_{1} \ldots \beta_{n}\right)$ is an analytical function of all its arguments which satisfies the requirement:

$$
f\left(\beta_{1} \ldots \beta_{n-1}, \beta_{n}+2 \pi i\right)=f\left(\beta_{n}, \beta_{1} \ldots \beta_{n-1}\right) P_{12} P_{23} \ldots P_{n 1} c_{n} c_{n}^{t} .
$$

3. $f\left(\beta_{1} \ldots \beta_{n}\right)$ has simple poles at the points $\beta_{j}=\beta_{i}+\pi i$ for $j>i$. The residue at the point $\beta_{n}=\beta_{n-1}+\pi i$ is given by

$$
\begin{aligned}
\underset{\beta_{n}=\beta_{n-1}+\pi i}{\operatorname{res}} f\left(\beta_{1} \ldots \beta_{n-1}, \beta_{n}\right)=f\left(\beta_{1} \ldots \beta_{n-2}\right) \otimes s_{n-1, n} \\
\quad \times\left(I-S_{n-1,1}\left(\beta_{n-1}-\beta_{1}\right) \ldots S_{n-1, n-2}\left(\beta_{n-1}-\beta_{n-2}\right)\right),
\end{aligned}
$$

where $s_{12}=c^{i_{1} i_{2}} e_{i_{1}} \otimes e_{i_{2}}\left(e_{i_{\mathrm{k}}}\right.$ is the base in $k^{\text {th }}$ space). The form factor $f\left(\beta_{1} \ldots \beta_{n}\right)$ has no other singularities in the strip $0<\operatorname{Im} \beta_{n}<2 \pi$ if there are no bound states in the theory.

Consider two operators $O_{1}, O_{2}$ with form factors $f_{1}, f_{2}$. Let us recall the crucial point of the proof of locality [15]. Consider the commutator $\left[O_{1}(x), O_{2}(y)\right]$ for $x<y$ ( $x, y$ are space variables). The vanishing of the commutator is equivalent essentially to the following fact. The product of form factors

$$
f_{1}\left(A_{1} \mid C B_{1}\right) f_{2}\left(C A_{2} \mid B_{2}\right)
$$

$\left(A_{1}, A_{2}, B_{1}, B_{2}\right.$ are some sets of rapidities) can be continued analytically with respect to the variable $\sigma=\sum_{\gamma \in C} \gamma$ by $-\pi i$ giving as a result the following product:

$$
f_{2}\left(A_{2} \mid B_{2} C\right) f_{1}\left(A_{1} C \mid B_{1}\right) \text {. }
$$


This fact follows from the relations (2.4)-(2.6). Really, using (2.4)-(2.6) one can show that the representation (2.3) is equivalent to the following:

$$
f\left(\alpha_{m} \ldots \alpha_{1} \mid \beta_{1} \ldots \beta_{n}\right)=c_{1}^{t} \ldots c_{m}^{t} f^{t_{1} \ldots t_{m}}\left(\beta_{1} \ldots \beta_{n}, \alpha_{m}+\pi i, \ldots \alpha_{1}+\pi i\right) .
$$

Using the formula (2.3) for $f_{1}$ and the formula (2.9) for $f_{2}$ one easily proves that (2.7) is the result of analytic continuation of (2.8).

In this paper we concentrate our attention on the energy momentum tensor $T_{\mu \nu}$ and parafermions which are closely connected with $T_{\mu v}$. It is convenient to use light cone components of the energy momentum tensor: $T_{\sigma \tau}(\sigma, \tau= \pm)$. Evidently the formfactors of $T_{\sigma \tau}$ can be presented as follows:

$$
f_{\sigma \tau}\left(\beta_{1} \ldots \beta_{n}\right)=\left(\sum_{j=1}^{n} M e^{\sigma \beta_{j}}\right)\left(\sum_{j=1}^{n} M e^{\tau \beta_{j}}\right) g\left(\beta_{1} \ldots \beta_{n}\right),
$$

where $M$ is the mass of the particle. Due to $T_{\sigma \tau}$ being a $(1,1)$ Lorenz tensor the function $g$ satisfies the equation:

$$
g\left(\beta_{1}+\delta, \ldots, \beta_{n}+\delta\right)=g\left(\beta_{1} \ldots \beta_{n}\right) .
$$

If the theory under consideration leads to some conformal field theory in scaling limit the function $g$ should satisfy the requirements:

1. The integral

$$
I_{n}=\int_{\beta_{n}>\beta_{n-1}>\ldots>\beta_{1}} d \beta_{2} \ldots d \beta_{n}\left\|g\left(\beta_{1} \ldots \beta_{n}\right)\right\|^{2}
$$

is convergent.

2. The series

$$
\sum_{n=0}^{\infty} I_{n}=c
$$

are convergent. The constant $c$ coincide with the central charge of the limit conformal field theory.

Consider a more general situation. Suppose the form factors of some local or quasilocal operator can be presented in a form

$$
f\left(\beta_{1} \ldots \beta_{n}\right)=\left(\sum M e^{\beta_{j}}\right)^{\Delta} h\left(\beta_{1} \ldots \beta_{n}\right),
$$

where $h$ satisfies the requirements similar to (2.12)-(2.13). Then the formfactors $f$ define some parafermion operator $\psi$ which becomes parafermion current [17] with dimensions $(\Delta, 0)$ in the scaling limit. This statement is quite obvious.

This is all the necessary general information in this paper about local operators in massive integrable models.

\section{Quantum Group Invariance and Parafermions}

In this section we shall show that quantum group invariance of integrable models implies the existence of parafermion operators which possess generalized statistics. Quantum group here is understood as it has been explained in Sect. 1. Usual 
groups can be considered as particular examples of quantum ones. We still consider the models with one particle and no bound states.

Suppose the $S$-matrix of the model has the asymptotics:

$$
S_{12}(\beta) \underset{\beta \rightarrow \infty}{\longrightarrow} R_{12}, \quad S_{12}(\beta) \underset{\beta \rightarrow-\infty}{\longrightarrow}\left(R_{21}\right)^{-1},
$$

where $R_{12}$ is a certain constant solution of Yang-Baxter equations. As it has been said in the Introduction if $R_{12}$ is not equal to 1 we deal really with particles possessing generalized statistics.

Suppose that it is possible to associate some quantum groups $G$ with the matrix $R$ :

$$
R_{12} g_{1} g_{2}=g_{2} g_{1} R_{12} .
$$

Suppose now that not only $R_{12}$ and $\left(R_{21}\right)^{-1}$ but $S_{12}(\beta)$ for arbitrary $\beta$ intertwine $g_{1}, g_{2}$ :

$$
S_{12}(\beta) g_{1} g_{2}=g_{2} g_{1} S_{12}(\beta) .
$$

If Eq. (3.1) holds we say that the $S$-matrix is invariant under the quantum group $G$. Evidently Eq. (3.1) generalizes the invariance under the usual isotopic group.

We suppose also that the antipode in $G$ is given by

$$
s(g)=c g^{t} c,
$$

where $c$ is the matrix of charge conjugation associated with the $S$-matrix. The properties (2.1) of the $S$-matrix mean that

$$
R_{12}^{-1}=c_{1} R_{12}^{t_{1}} c_{1} .
$$

Together with (1.2) Eq. (3.3) shows that the matrix is correctly normalized for the representations

$$
\varrho_{2}^{+}\left(g_{1}\right)=R_{12}, \quad \varrho_{2}^{-}\left(g_{1}\right)=\left(R_{21}\right)^{-1}
$$

being true representations of Hopf algebra $A$.

Now, let us define the action of quantum group $G$ in the space of states of the model. More precisely, we define the mapping $T_{g}: H \rightarrow H \otimes A(H$ is the space of states):

$$
\begin{aligned}
& T_{g}\left|\beta_{n} \ldots \beta_{1}\right\rangle=\left|\beta_{n} \ldots \beta_{1}\right\rangle g_{n} \ldots g_{1} \\
& \left\langle\beta_{1} \ldots \beta_{n}\right| T_{g}=g_{1} \ldots g_{n}\left\langle\beta_{1} \ldots \beta_{n}\right|
\end{aligned}
$$

as usual $g_{i}$ denotes $g$ acting in $i^{\text {th }}$ isotopic space. Equation (3.1) means that scattering commutes with $T_{g}$ :

$$
T_{g} \mathscr{S}=\mathscr{S} T_{g},
$$

where $\mathscr{S}$ is the full $S$-matrix defined as an operator in the space of states. Thus we have a symmetry of the theory. Certainly this symmetry is unusual and it is not clear that we shall be able to get something from it. Really, the operator $T_{g}$ does not act in the space of states but maps it into the tensor product of $H$ with some mysterious $A$. However, the reasonings of Sect. 1 show that it is possible to describe in that way the action of the usual isotopic group identifying $A$ with the 
algebra of functions on the group. So, one can hope that noncommutative generalization of $A$ also can be useful. As it has been shown in the paper [13] for SG model that is the case and the quantum group invariance may be even more restrictive than the usual one.

Let us turn now to the local operators. The symmetry with respect to the quantum group is really interesting if it can be spread to off-shell objects, otherwise it can be regarded as an occasional on-shell property. In particular it is important to understand if the energy-momentum tensor is invariant under the action of the quantum group. The invariance of $T_{\mu \nu}$ means that

$$
T_{g} T_{\mu \nu}(x)=T_{\mu \nu}(x) T_{g} .
$$

This equation is equivalent to the following properties of matrix elements:

$$
g\left(\alpha_{k} \ldots \alpha_{1} \mid \beta_{1} \ldots \beta_{m}\right)=s\left(g_{m+1}\right) \ldots s\left(g_{m+k}\right) g\left(\alpha_{k} \ldots \alpha_{1} \mid \beta_{1} \ldots \beta_{m}\right) g_{m} \ldots g_{1},
$$

where the spaces associated with $\alpha_{1} \ldots \alpha_{k}$ have the numbers $m+1, \ldots m+k$. Using $(3.2,2.3)$ one easily makes sure that Eqs. (3.5) follow from the following one:

$$
g\left(\beta_{1} \ldots \beta_{n}\right)=g\left(\beta_{1} \ldots \beta_{n}\right) g_{n} \ldots g_{1} .
$$

Let us discuss this condition. Its left-hand side is a vector in the tensor product of isotopic spaces, while the right-hand side is the vector in the tensor product whose components lie in $A$. Thus Eq. (3.6) seems strange. Recall, however, the condition

$$
s(g) g=g s(g)=I .
$$

The consistency of this condition with another Hopf algebra conditions means that $s(g) g=g s(g)$ belongs to the center of the Hopf algebra $A$, and we fix the value of the central element by (3.7). The bilinear center element $s(g) g$ generates many center elements which are homogeneous functions of $g$ of $n^{\text {th }}$ degree. Equation (3.6) means that all the components of the right-hand side vector belong to the center of $A$, their values are identified with $\mathbb{C}$-numbers due to (3.7), and these $\mathbb{C}$-number components coincide with the components of the left-hand side. It is instructive to consider the usual isotopic group from this point of view. We suppose that the theory contains no bound states. In this case only even particle formfactors $T_{\mu \nu}$ differ from zero. This fact is in agreement with Eq. (3.7) because the center of $A$ generated by $s(g) g$ can contain only homogeneous in $g$ functions of even degree.

Suppose that Eq. (3.6) does take place. Then the theory is called quantum group invariant. Our next goal is to show that if some additional assumptions are made then the quantum group invariance implies the existence of parafermion operators possessing generalized statistics.

The convergence of the integral

$$
\int\left\|g\left(\beta_{1} \ldots \beta_{n}\right)\right\|^{2} d \beta_{2} \ldots d \beta_{n}
$$

means that $g\left(\beta_{1} \ldots \beta_{n}\right)$ decreases when $\beta_{n} \rightarrow \infty$. We suppose that it decreases exponentially:

$$
g\left(\beta_{1} \ldots \beta_{n}\right) \underset{\beta_{n} \rightarrow \infty}{\sim} e^{-\Delta \beta_{n}} f\left(\beta_{1} \ldots \beta_{n-1}\right),
$$

where $\Delta$ is a certain positive number, $f$ belongs to the tensor product of the isotopic spaces associated with $\beta_{1} \ldots \beta_{n}$. The function $f\left(\beta_{1} \ldots \beta_{n-1}\right)$ does not 
depend on $\beta_{n}$, that is why it is convenient to extract explicitly the isotopic index corresponding to the $n^{\text {th }}$ space: $f_{i}\left(\beta_{1} \ldots \beta_{n-1}\right)$. The functions $f_{i}$ can be considered as matrix elements of vector operator $\psi_{i}(x)$ :

$$
\left\langle 0\left|\psi_{i}(0,0)\right| \beta_{n-1} \ldots \beta_{1}\right\rangle=f_{i}\left(\beta_{1} \ldots \beta_{n-1}\right) .
$$

Relation (2.11) implies that

$$
f_{i}\left(\beta_{1} \ldots \beta_{k}\right)=\left(\sum M e^{\beta_{j}}\right)^{4} h\left(\beta_{1} \ldots \beta_{k}\right),
$$

where

$$
h_{i}\left(\beta_{1}+\delta, \ldots, \beta_{k}+\delta\right)=h_{i}\left(\beta_{1} \ldots \beta_{k}\right) .
$$

The convergence of the integral (2.12) implies the convergence of

$$
\int\left\|h_{i}\left(\beta_{1} \ldots \beta_{k}\right)\right\|^{2} d \beta_{2} \ldots d \beta_{k} .
$$

Thus the operator $\psi_{i}$ is a parafermionic operator and we have to investigate its locality.

We define the general matrix elements of $\psi_{i}$ by the formula similar to (2.3):

$$
f_{i}\left(\alpha_{k} \ldots \alpha_{1} \mid \beta_{1} \ldots \beta_{m}\right)=c_{k} \ldots c_{1} f_{i}^{t_{k} \pm t_{1}}\left(\alpha_{k}-\pi i, \ldots, \alpha_{1}-\pi i, \beta_{1} \ldots \beta_{m}\right) .
$$

For local operators the definition (2.3) is equivalent to the following one (2.9):

$$
f_{i}\left(\alpha_{k} \ldots \alpha_{1} \mid \beta_{1} \ldots \beta_{m}\right)=c_{k}^{t} \ldots c_{1}^{t} f_{i}^{t_{k} \ldots t_{1}}\left(\beta_{1} \ldots \beta_{m}, \alpha_{k}+\pi i, \ldots, \alpha_{1}+\pi i\right)
$$

due to Eq. (2.5). In our case the functions $f$ and $f$ differ. Using Eqs. (2.5) and the definition (3.8) one gets

$$
f_{a}\left(\alpha_{k} \ldots \alpha_{1} \mid \beta_{1} \ldots \beta_{n}\right)=f_{a}\left(\alpha_{k} \ldots \alpha_{1} \mid \beta_{1} \ldots \beta_{n}\right) R_{n a} \ldots R_{1 a},
$$

where $R_{i a}$ act in isotopic spaces associated with $\beta_{i}$, the functions $f_{i}$ are combined into the covector $f_{a}$ from the space with "number" $a$.

So we have two operators $\psi_{a}, \tilde{\varphi}_{a}$ with the form factors $f_{a}, \tilde{f}_{a}$. These operators are related by

$$
\tilde{\psi}_{a}(x)=\psi_{a}(x) T_{e_{a}^{+}(g)} .
$$

Consider the commutator $\left[\psi_{a}(x), \psi_{b}(y)\right]$ for $x<y$ ( $x, y$ are space variables). The form factors of $\psi_{a}$ and $\tilde{\psi}_{b}$ are constructed from $f$ using the formulas (3.9) and (3.10) respectively. This fact implies that the commutator $\left[\psi_{a}(x), \tilde{\psi}_{b}(y)\right]$ for $x<y$ is equal to zero (see the explanations given in Sect. 2 concerning the proof of the locality).

Thus

$$
\psi_{a}(x) \tilde{\psi}_{b}(y)=\tilde{\psi}_{b}(y) \psi_{a}(x), \quad x<y .
$$

Now using Eq. (3.11) and the equation

$$
T_{g} \psi_{a}=\psi_{a} g_{a} T_{g},
$$

which follows from the definition (3.8) one gets

$$
\psi_{a}(x) \psi_{b}(y)=\psi_{b}(y) \psi_{a}(x) \varrho_{b}^{+}\left(g_{a}\right)=\psi_{b}(y) \psi_{a}(x) R_{a b}, \quad x<y .
$$


In indices this relation can be rewritten as

$$
\psi_{i}(x) \psi_{j}(y)=\psi_{\ell}(y) \psi_{k}(x) R_{i j}^{k \ell}, \quad x<y .
$$

Thus the operator $\psi$ does possess generalized statistics.

In the same fashion one can consider the operator $\bar{\psi}_{i}$ whose matrix elements are defined by (3.10) through the functions $\bar{f}_{i}$ :

$$
g\left(\beta_{1} \ldots \beta_{n}\right) \underset{\beta_{n} \rightarrow-\infty}{\longrightarrow} e^{\Delta \beta_{n}} \bar{f}\left(\beta_{1} \ldots \beta_{n-1}\right) .
$$

It can be shown that $\bar{\psi}_{i}$ satisfies the relation

$$
\bar{\psi}_{i}(x) \bar{\psi}_{j}(y)=\bar{\psi}_{\ell}(y) \bar{\psi}_{k}(x) R_{i j}^{k \ell}, \quad x>y .
$$

The commutation relation of $\psi_{i}(x)$ and $\bar{\psi}_{j}(y)$ does not depend on the position of $x$ and $y$ :

$$
\psi_{i}(x) \bar{\psi}_{j}(y)=\bar{\psi}_{\ell}(y) \psi_{k}(x) R_{i j}^{k \ell}
$$

Certainly the operators $\psi_{i}, \bar{\psi}_{i}$ are local with respect to $T_{\mu \nu}$ :

$$
\left[\psi_{i}(x), T_{\mu \nu}(y)\right]=\left[\bar{\psi}_{i}(x), T_{\mu v}(y)\right]=0 .
$$

Thus we have shown that the quantum group invariance together with some additional requirements implies the existence of parafermion operators satisfying the generalized statistics. In the next two sections examples will be considered.

\section{Parafermions in SG and RSG(r) Model}

Let us describe briefly the results of the paper [13]. Consider SG model with the Lagrangian

$$
\mathscr{L}=\int\left(\frac{1}{2}\left(\partial_{\mu} \varphi\right)^{2}+m^{2} \cos (\sqrt{\gamma} \varphi)\right) d x
$$

We shall use the renormalized coupling constant $\xi=\frac{\gamma}{8 \pi-\gamma}$. For $\xi>\pi$ the spectrum of the model contains only one two-component particle (soliton) [22]. Let us consider the following base in the space of states and the conjugated space:

$$
\begin{gathered}
\left|\beta_{n} \ldots \beta_{1}\right\rangle=\left|\beta_{n} \ldots \beta_{1}\right\rangle_{\mathrm{SG}} \exp \left(-\frac{\pi}{2 \xi} \sigma_{1}^{3} \beta_{1}\right) \ldots \exp \left(-\frac{\pi}{2 \xi} \sigma_{n}^{3} \beta_{n}\right), \\
\left\langle\beta_{1} \ldots \beta_{n}\right|=\exp \left(\frac{\pi}{2 \xi} \sigma_{1}^{3} \beta_{1}\right) \ldots \exp \left(\frac{\pi}{2 \xi} \sigma_{n}^{3} \beta_{n}\right)\left\langle\left.\beta_{1} \ldots \beta_{n}\right|_{\mathrm{SG}},\right.
\end{gathered}
$$

where $\left|\beta_{n} \ldots \beta_{1}\right\rangle_{\mathrm{SG}}$ are normalized $n$-soliton states, $\sigma_{i}^{3}$ is the Pauli matrix $\sigma^{3}$ acting in the isotopic space associated with the $i^{\text {th }}$ soliton. Evidently the bases $\left|\beta_{n} \ldots \beta_{1}\right\rangle$, $\left\langle\beta_{1} \ldots \beta_{n}\right|$ are dual bases:

$$
\begin{gathered}
\left\langle\alpha_{1} \ldots \alpha_{m} \mid \beta_{n} \ldots \beta_{1}\right\rangle=\delta_{m n} \prod \delta\left(\alpha_{i}-\beta_{i}\right) I, \\
\alpha_{m}>\ldots>\alpha_{1}, \beta_{n}>\ldots>\beta_{1},
\end{gathered}
$$

but they are not conjugated with respect to $\mathrm{SG}$ conjugation:

$$
\left|\beta_{n} \ldots \beta_{1}\right\rangle_{\mathrm{SG}}^{*}=\left\langle\left.\beta_{1} \ldots \beta_{n}\right|_{\mathrm{SG}}\right. \text {. }
$$


The two-particle $S$-matrix written in the base $\left|\beta_{n} \ldots \beta_{1}\right\rangle,\left\langle\beta_{1} \ldots \beta_{n}\right|$ is

$$
S_{12}(\beta)=\frac{S_{0}(\beta)}{\operatorname{sh} \frac{\pi}{\xi}(\beta-\pi i)}\left(\begin{array}{c}
\operatorname{sh} \frac{\pi}{\xi}(\beta-\pi i) \\
-\operatorname{sh} \frac{\pi}{\xi} \beta,-\operatorname{sh} \frac{\pi^{2} i}{\xi} e^{\frac{\pi}{\xi} \beta} \\
-\operatorname{sh} \frac{\pi^{2} i}{\xi} e^{-\frac{\pi}{\xi} \beta},-\operatorname{sh} \frac{\pi}{\xi} \beta \\
\operatorname{sh} \frac{\pi}{\xi}(\beta-\pi i)
\end{array}\right)
$$

Rewrite the $S$-matrix in the form:

$$
S_{12}(\beta)=\frac{S_{0}(\beta)}{\operatorname{sh} \frac{\pi}{\xi}(\beta-\pi i)}\left(e^{\frac{\pi}{\xi} \beta} q^{-1 / 4} R_{12}(q)-e^{-\frac{\pi}{\xi} \beta} q^{1 / 4}\left(R_{21}(q)\right)^{-1}\right),
$$

where $R_{12}(q)$ is given by (1.4), $q=\exp \left(\frac{2 \pi^{2} i}{\xi}\right), q^{1 / 2}=-\exp \left(\frac{\pi^{2} i}{\xi}\right)$. Let us introduce the quantum group $S L(2)_{q}$. The $S$-matrix is evidently $S L(2)_{q}$ invariant:

$$
S_{12}(\beta) g_{1} g_{2}=g_{2} g_{1} S_{12}(\beta) \text {. }
$$

The $S$-matrix possesses the asymptotics

$$
S_{12}(\beta) \underset{\beta \rightarrow \infty}{\longrightarrow} R_{12}, S_{12}(\beta) \underset{\beta \rightarrow-\infty}{\longrightarrow}\left(R_{21}\right)^{-1} .
$$

Thus the scattering in SG satisfies all the requirements of Sect. 3 .

The form factors of local operators for SG were calculated in [14]. Using the explicit formulas for the form factors it has been shown in [13] that the modified energy-momentum tensor

$$
\widetilde{T}_{\mu \nu}=T_{\mu \nu}+i 2^{-5 / 2} \frac{\sqrt{\gamma}}{\xi} \varepsilon_{\mu \mu^{\prime}} \varepsilon_{\nu \nu^{\prime}} \partial_{\mu^{\prime}} \partial_{v^{\prime}} \varphi
$$

is invariant under the action of quantum group. Trace of $\widetilde{T}_{\mu \nu}$ is equal to $m \exp (i \sqrt{\gamma} \varphi)$. The central charge of $\widetilde{T}_{\mu \nu}$ in the scaling limit is equal to

$$
c=1-6\left(\frac{\gamma}{8 \pi}+\frac{8 \pi}{\gamma}-2\right) \text {. }
$$

We would not like to write down the explicit formulas for the form factors of $\widetilde{T}_{\mu \nu}$ which can be found in [14] (certainly, one has to rewrite these formulas in the base $\left|\beta_{n} \ldots \beta_{1}\right\rangle$ which differs from $\left.\left|\beta_{n} \ldots \beta_{1}\right\rangle_{\mathrm{SG}}(4.1)\right]$. The important point concerning 
these form factors is that the functions $g$ associated with them (4.1) satisfies the following asymptotics:

$$
\begin{aligned}
& g\left(\beta_{1} \ldots \beta_{n}\right) \underset{\beta_{n} \rightarrow \infty}{\longrightarrow} \exp \left(-\Delta \beta_{n}\right) f\left(\beta_{1} \ldots \beta_{n-1}\right), \\
& g\left(\beta_{1} \ldots \beta_{n}\right) \underset{\beta_{n} \rightarrow-\infty}{\longrightarrow} \exp \left(\Delta \beta_{n}\right) \bar{f}\left(\beta_{1} \ldots \beta_{n-1}\right),
\end{aligned}
$$

where

$$
\Delta=\frac{1}{4}+\frac{3 \pi}{4 \xi} .
$$

The explicit formulas for $f$ and $\bar{f}$ which can be obtained from the explicit formulas for $g$ will be presented later. Before doing that we would like to discuss some principal points.

The functions $f, \bar{f}$ define two parafermionic operators $\bar{\psi}, \psi$ as it has been explained in Sect. 3. The operators $\psi, \bar{\psi}$ are two-component ones, they satisfy the following equal time commutation relations:

$$
\begin{aligned}
& \psi_{i}(x) \psi_{j}(y)=\psi_{\ell}(y) \psi_{k}(x) R_{i j}^{k \ell}, x<y ; \\
& \bar{\psi}_{i}(x) \bar{\psi}_{j}(y)=\bar{\psi}_{\ell}(y) \bar{\psi}_{k}(x) R_{i j}^{k \ell}, \quad x>y ; \\
& \psi_{i}(x) \bar{\psi}_{j}(y)=\bar{\psi}_{\ell}(y) \psi_{k}(x) R_{i j}^{k \ell}, \quad \forall x, y .
\end{aligned}
$$

The operators $\psi, \bar{\psi}$ are local with respect to $\widetilde{T}_{\mu \nu}$. It is necessary to emphasize, however, that the operators $\psi, \bar{\psi}$ are very bad with respect to those operators whose transformation properties under the action of $S L(2)_{q}$ are not property defined. For example, the SG energy-momentum tensor (not modified) $T_{\mu \nu}$ is an infinite sum of nonlocal operators which transform under different irreducible representations os $S L(2)_{q}$. That is why the commutation relations of $\psi, \bar{\psi}$, and $T_{\mu \nu}$ cannot be written in closed form.

The space of states and the dual space can be decomposed into direct sums:

$$
H=\bigoplus_{j=0}^{\infty} H_{j}, \quad H^{\prime}=\bigoplus_{j=0}^{\infty} H_{j}^{\prime}
$$

( $j$ is half-integer). The subspaces $H_{j}, H_{j}^{\prime}$ are spin $j$ subspaces with respect to $S L(2)_{q}$. The bases in $H_{j}, H_{j}^{\prime}$ can be constructed as follows:

$$
\begin{aligned}
\left|j, \beta_{n}, a_{n-1}, \beta_{n-1} \ldots a_{1}, \beta_{1}\right\rangle_{m}=\left|\beta_{n} \ldots \beta_{1}\right\rangle_{i_{n} \ldots i_{1}} E_{m}^{i_{n} \ldots i_{1}}\left(j, a_{n-1} \ldots a_{1}\right), \\
{ }^{m}\left\langle\beta_{1}, a_{1}, \beta_{2} \ldots a_{n-1}, \beta_{n}, j\right|={ }^{i_{1} \ldots i_{n}}\left\langle\beta_{1} \ldots \beta_{n}\right| E_{i_{1} \ldots i_{n}}^{m}\left(a_{1} \ldots a_{n-1}, j\right), \\
E_{m}^{i_{n} \ldots i_{1}}\left(j, a_{n-1}, \ldots, a_{1}\right)=E_{i_{1} \ldots i_{n}}^{m}\left(a_{1} \ldots a_{n-1}, j\right) \\
=\left[\begin{array}{ccc}
a_{n-1} & \frac{1}{2} & j \\
m_{n-1} & i_{n} & m
\end{array}\right]_{q}^{\prime}\left[\begin{array}{ccc}
a_{n-2} & \frac{1}{2} & a_{n-1} \\
m_{n-2} & i_{n-1} & m_{n-1}
\end{array}\right]_{q}^{\prime} \times \ldots \\
\times\left[\begin{array}{ccc}
0 & \frac{1}{2} & a_{1} \\
0 & i_{1} & m_{1}
\end{array}\right]_{q}^{\prime}, \quad m_{j}=i_{j}+m_{j-1},
\end{aligned}
$$

where $\left[\begin{array}{lll}a_{1} & a_{2} & a_{3} \\ m_{1} & m_{2} & m_{3}\end{array}\right]_{q}^{\prime}$ are $q$-analogs of Clebsch-Gordan coefficients (CGC) [23]. 
For $a_{2}=\frac{1}{2}$ they are given by the formulas:

$$
\begin{aligned}
& {\left[\begin{array}{ccc}
j & \frac{1}{2} & j+\frac{1}{2} \\
m & \frac{1}{2} & m+\frac{1}{2}
\end{array}\right]_{q}^{\prime}=q^{-\frac{1}{4}(m+j+1)},} \\
& {\left[\begin{array}{ccc}
j & \frac{1}{2} & j-\frac{1}{2} \\
m & \frac{1}{2} & m+\frac{1}{2}
\end{array}\right]_{q}^{\prime}=-[j-m] q^{-\frac{1}{4}(j+m)},} \\
& {\left[\begin{array}{ccc}
j & \frac{1}{2} & j+\frac{1}{2} \\
m & -\frac{1}{2} & m-\frac{1}{2}
\end{array}\right]_{q}^{\prime}=q^{\frac{1}{4}(j-m+1)},} \\
& {\left[\begin{array}{ccc}
j & \frac{1}{2} & j-\frac{1}{2} \\
m & -\frac{1}{2} & m-\frac{1}{2}
\end{array}\right]_{q}^{\prime}=q^{\frac{1}{4}(j+m)}[j+m],}
\end{aligned}
$$

where

$$
[n]=\frac{q^{n / 2}-q^{-n / 2}}{q^{1 / 2}-q^{-1 / 2}}, \quad[0] \equiv 1,
$$

the normalization of CGC differs from that used in [23] in order that they are Laurent polynomials of $q^{1 / 4}$. The following formula for the pairings of the vectors (4.3) holds:

$$
\begin{aligned}
& m^{\prime}\left\langle\beta_{1}^{\prime}, a_{1}^{\prime}, \beta_{2}^{\prime} \ldots \beta_{n^{\prime}}^{\prime}, j^{\prime} \mid j, \beta_{n}, a_{n-1} \ldots a_{1} \beta_{1}\right\rangle_{m} \\
& =\delta_{n n^{\prime}} \delta_{m}^{m^{\prime}} \delta_{j j^{\prime}} \frac{1}{[j-m] ![j+m] !} \prod_{k=1}^{n-1}\left[2 a_{k}+1\right] \prod \delta\left(\beta_{i}-\beta_{i}^{\prime}\right), \\
& \beta_{n}>\ldots>\beta_{1}, \beta_{n}^{\prime}>\ldots>\beta_{1}^{\prime}
\end{aligned}
$$

which follows from the formula:

$$
\begin{aligned}
& E_{m}\left(j, a_{n-1} \ldots a_{1}\right) E^{m^{\prime}}\left(a_{1}^{\prime} \ldots a_{n-1}^{\prime}, j^{\prime}\right) \\
& \quad=\delta_{j j^{\prime}} \prod \delta_{a_{k} a_{k}^{\prime}} \prod\left[2 a_{k}+1\right] \frac{1}{[j+m] ![j-m] !} .
\end{aligned}
$$

Consider now the operators ${ }_{j_{2}} \psi_{j_{1}},{ }_{j_{2}} \bar{\psi}_{j_{1}}$ which provide the block decomposition of $\psi, \bar{\psi}$ with respect to the decomposition $H=\oplus H_{j}$ :

$$
\begin{aligned}
& j_{2} \psi_{j_{1}}(x)=\sum_{i=1}^{2} \psi_{i}(x)\left[\begin{array}{ccc}
j_{1} & \frac{1}{2} & j_{2} \\
\cdot & i & \cdot
\end{array}\right]_{q}^{\prime}, \\
& j_{2} \bar{\psi}_{j_{1}}(x)=\sum_{i=1}^{2} \psi_{i}(x)\left[\begin{array}{ccc}
j_{1} & \frac{1}{2} & j_{2} \\
\cdot & i & \cdot
\end{array}\right]_{q}^{\prime},
\end{aligned}
$$

where dots under $j_{1}, j_{2}$ correspond to indices $m_{1}, m_{2}$ in the spaces $H_{j_{1}}, H_{j_{2}}$. The operators ${ }_{j_{2}} \psi_{j_{1}}, j_{2} \bar{\psi}_{j_{1}}$ satisfy the following same time commutation relations:

$$
\begin{aligned}
& j_{3} \psi_{j_{2}}(x)_{j_{2}} \psi_{j_{1}}(y)=\sum_{j_{2}^{\prime}} B\left(j_{3}, j_{1}, j_{2}, j_{2}^{\prime}\right)_{j_{3}} \psi_{j_{2}^{\prime}}(y)_{j_{2}} \psi_{j_{1}}(x), x<y \\
& j_{3} \bar{\psi}_{j_{2}}(x)_{j_{2}} \bar{\psi}_{j_{1}}(y)=\sum_{j_{2}^{\prime}} B\left(j_{3}, j_{1}, j_{2}, j_{2}^{\prime}\right)_{j_{3}} \bar{\psi}_{j_{2}^{\prime}}(y)_{j_{2}} \bar{\psi}_{j_{1}}(x), x>y ; \\
& j_{3} \psi_{j_{2}}(x)_{j_{2}} \bar{\psi}_{j_{1}}(y)=\sum_{j_{2}^{\prime}} B\left(j_{3}, j_{1}, j_{2}, j_{2}^{\prime}\right)_{j_{3}} \bar{\psi}_{j_{2}}(y)_{j_{2}} \psi_{j_{1}}(x), \forall x, y,
\end{aligned}
$$


where the coefficients $B$ are connected with $q$-analogs of $6 j$-symbols [23]:

$$
\begin{gathered}
B\left(j_{3}, j_{1}, j_{2}, j_{2}\right)=q^{c_{j_{1}}+c_{j_{3}}-c_{j_{2}}-c_{j^{\prime}}}\left\{\begin{array}{lll}
\frac{1}{2} & j_{1} & j_{2}^{\prime} \\
\frac{1}{2} & j_{3} & j_{2}
\end{array}\right\}_{q}^{\prime}, \\
c_{j}=j(j+1) .
\end{gathered}
$$

For the normalization of CGC accepted in this paper the $6 j$ symbols are equal to:

$$
\begin{aligned}
& \left\{\begin{array}{lll}
\frac{1}{2} & j & j-\frac{1}{2} \\
\frac{1}{2} & j & j+\frac{1}{2}
\end{array}\right\}_{q}^{\prime}=-\frac{[2 j]}{[2 j+1]}, \quad\left\{\begin{array}{lll}
\frac{1}{2} & j & j+\frac{1}{2} \\
\frac{1}{2} & j & j-\frac{1}{2}
\end{array}\right\}_{q}^{\prime}=-\frac{[2 j+2]}{[2 j+1]}, \\
& \left\{\begin{array}{lll}
\frac{1}{2} & j & j-\frac{1}{2} \\
\frac{1}{2} & j & j-\frac{1}{2}
\end{array}\right\}_{q}^{\prime}=-\frac{1}{[2 j+1]}, \quad\left\{\begin{array}{lll}
\frac{1}{2} & j & j+\frac{1}{2} \\
\frac{1}{2} & j & j+\frac{1}{2}
\end{array}\right\}_{q}^{\prime}=\frac{1}{[2 j+1]}, \\
& \left\{\begin{array}{ccc}
\frac{1}{2} & j & j+\frac{1}{2} \\
\frac{1}{2} & j+1 & j+\frac{1}{2}
\end{array}\right\}_{q}^{\prime}=\left\{\begin{array}{ccc}
\frac{1}{2} & j & j-\frac{1}{2} \\
\frac{1}{2} & j-1 & j-\frac{1}{2}
\end{array}\right\}_{q}^{\prime}=1 .
\end{aligned}
$$

Consider now the case of rational coupling constants: $\xi=\frac{\pi r}{r_{1}}$. For these coupling constants $q^{r}=1$. From the formula (4.4) it follows that

$$
E_{m}\left(j, a_{n-1}, \ldots a_{1}\right) E^{m}\left(a_{1} \ldots a_{n-1}, j\right)=0
$$

if $j \leqq \frac{r-2}{2}$, but some of intermediate spins $a_{i}$ exceed $\frac{r-2}{2}$. This is very important point which was recognized in the paper [13]. This fact provides the possibility of reduction of SG to $\operatorname{RSG}\left(r / r_{1}\right)$ for $\xi=\frac{\pi r}{r_{1}}[13]$.

Consider the Green function of the kind:

$$
\left\langle\left. 0\right|_{0} \psi_{j_{n-1}}\left(x_{n}\right) \ldots j_{1} \psi_{0}\left(x_{1}\right) \mid 0\right\rangle \text {. }
$$

By the definition this Green function can be presented as follows

$$
\left\langle 0\left|\psi_{i_{n}}\left(x_{n}\right) \ldots \psi_{i_{1}}\left(x_{1}\right)\right| 0\right\rangle E_{0}^{i_{1} \ldots i_{n}}\left(0, j_{n-1}, j_{n-2} \ldots j_{1}, 0\right) .
$$

Due to the equations

$$
\begin{gathered}
T_{g}|0\rangle=|0\rangle,\langle 0| T_{g}=\langle 0|, \\
T_{g} \psi_{i}(x)=\psi_{j}(x) g_{i}^{j} T_{g}
\end{gathered}
$$

the vector $\left\{\left\langle 0\left|\psi_{i_{n}}\left(x_{n}\right) \ldots \psi_{i_{1}}\left(x_{1}\right)\right| 0\right\rangle\right\}$ belongs to the singlet with respect to $S L(2)_{q}$ subspace of $\left(\mathbb{C}^{2}\right)^{\otimes n}$. Hence it can be decomposed with respect to the base $\left\{E_{i_{1} \ldots i_{n}}^{0}\left(0, a_{1}, \ldots a_{n-1}, 0\right)\right\}$. Now from Eq. (4.4) it follows that only those Green functions (4.7) differ from zero for which $j_{k} \leqq \frac{r-2}{2} \forall k$. Thus ${ }_{j_{2}} \psi_{j_{1}}$ are equal to zero in a weak sense if the Green functions of the type (4.7) are considered for $j_{1}$ or $j_{2}>\frac{r-2}{2}$. 
Generally, consider local or quasilocal operators which transform under the actions of $S L(2)_{q}$ via finite dimensional irreducible representations (the operators $\widetilde{T}_{\mu v}, \psi, \bar{\psi}$ are particular examples). Evidently, these operators constitute ${ }_{+}$closed operator algebra. An operator $O^{j}$ transforming with respect to $\operatorname{spin} j$ representation can be decomposed into blocks which act from $H_{j_{1}}$ to $H_{j_{2}}\left(\left|j_{1}-j\right| \leqq j_{2} \leqq j_{1}+j\right)$. Consider the Green function of these operators. Then the blocks ${ }_{j_{2}} O_{j_{1}}^{j_{1}}$ with $j_{1}, j_{2}$ $>\frac{r-2}{2}$ are effectively qual to zero.

What kind of intermediate states can appear in the Green functions (4.7) for $j_{k} \leqq \frac{r-2}{2}$ ? Evidently, the intermediate states are

$$
\left|a_{n}, \beta_{n}, a_{n-1} \ldots \beta_{1}\right\rangle,\left\langle\beta_{1}, a_{1}, \beta_{2}, \ldots a_{n-1}, \beta_{n}, a_{n}\right|
$$

with $a_{n} \leqq \frac{r-2}{2}$ [we combined the vectors (4.3) with different into one vector]. The formula (4.4) for the scalar products implies that

$$
\left\langle\beta_{1}^{\prime}, a_{1}^{\prime} \ldots \beta_{n}^{\prime}, a_{n}^{\prime} \mid a_{n}, \beta_{n} \ldots a_{1}, \beta_{1}\right\rangle=\delta_{n n^{\prime}} \prod \delta_{a_{i} a_{i}^{\prime}}^{n-1} \prod_{i=1}^{n-1}\left[2 a_{i}+1\right] \frac{1}{\left[2 a_{n}\right] !} .
$$

It follows from (4.8) that for $\xi=\frac{\pi r}{r_{1}}$ and $a_{n} \leqq \frac{r-2}{2}$ the vectors (4.3) with $a_{k}>\frac{r-2}{2}$ for some $k$ are "orthogonal to everything." That is why the space of states $H$ and the dual space $H^{\prime}$ can be effectively restricted to those generated by the vectors (4.3) with $a_{i} \leqq \frac{r-2}{2}$ (we denote the subspaces by $H_{r}, H_{r}^{\prime}$ ). The reduced theories which are denoted by RSG $\left(r / r_{1}\right)$ coincide with the perturbations of the CFT $M_{r, r+r_{1}}$ by the operator $\phi_{13}$. As it has been explained in [13] for the physical interpretation of the reduced models we have to introduce new Hermitian conjugation because SG one is not suitable. The natural receipt is to require

$$
\left|a_{n}, \beta_{n}, a_{n-1} \ldots \beta_{1}\right\rangle^{*}=\left\langle\beta_{1} \ldots a_{n-1}, \beta_{n}, a_{n}\right| \operatorname{sgn}\left(\prod_{i=1}^{n-1}\left[2 a_{i}+1\right] \frac{1}{\left[2 a_{n}\right] !}\right) .
$$

The $S$-matrix of RSG $\left(r / r_{1}\right)$ is SG $S$-matrix rewritten in the base (4.3), it is effectively RSOS $S$-matrix $[13,24]$. The $S$-matrix is unitary with respect to the conjugation (4.9) for $\xi=\frac{\pi r}{k r+1}, r \geqq 3, k=0 ; r \geqq 3, k \geqq 1 ; \xi=\frac{3 \pi}{3 k+2}, k \geqq 0$. The algebra of local operators is closed with respect to the conjugation only for $\xi=\pi r$ [13]. These values of coupling constant are of greatest interest because the corresponding reduced models (RSG $(r)$ ) coincide with the perturbations of the CFT models $M_{r}$ by the operator $\phi_{13}$. The above construction gives for RSG $(r)$ the operators ${ }_{j_{2}} \psi_{j_{1}}$, ${ }_{j_{2}} \bar{\psi}_{j_{1}}$ with $j_{1}, j_{2} \leqq \frac{r-2}{2}$ with generalized statistics (4.6). The operators ${ }_{j_{2}} \psi_{j_{1}},{ }_{j_{2}} \bar{\psi}_{j_{1}}$ are parafermionic operators which give in scaling limit the parafermionic currents with dimensions $(\Delta, 0),(0, \Delta), \Delta=\frac{1}{4}+\frac{3}{4 r}(4.2)$. This dimensions coincides with the dimension $\Delta_{21}$ for $M_{r}$. 
Now we give explicit formulas for the form factors of $\psi, \bar{\psi}$. They are obtained from the formfactors of $\widetilde{T}_{\mu \nu}[14]$. The form factors

$$
\begin{aligned}
& f\left(\beta_{1} \ldots \beta_{2 n+1}\right)=\left\langle 0|\psi(0,0)| \beta_{2 n+1} \ldots \beta_{1}\right\rangle, \\
& \bar{f}\left(\beta_{1} \ldots \beta_{2 n+1}\right)=\left\langle 0|\bar{\psi}(0,0)| \beta_{2 n+1} \ldots \beta_{1}\right\rangle
\end{aligned}
$$

are vectors from the tensor product of the isotopic spaces $\left(\mathbb{C}^{2}\right)^{\otimes 2 n+1}$. From the symmetry property

$$
\begin{aligned}
& f\left(\beta_{1} \ldots \beta_{i}, \beta_{i+1} \ldots \beta_{2 n+1}\right) S_{i i+1}\left(\beta_{i}-\beta_{i+1}\right) \\
& \quad=f\left(\beta_{1} \ldots \beta_{i+1}, \beta_{i} \ldots \beta_{2 n+1}\right) P_{i i+1}
\end{aligned}
$$

and the form of $S$-matrix it follows that it is sufficient to point out two components of the form factors:

$$
\begin{aligned}
& f\left(\beta_{1} \ldots \beta_{2 n+1}\right) \underbrace{1 \ldots 1}_{n+1} \underbrace{2 \ldots 2}_{n}, \\
& f\left(\beta_{1} \ldots \beta_{2 n+1}\right) \underbrace{2 \ldots 2}_{n+1} \underbrace{2 \ldots 1}_{n}
\end{aligned}
$$

(the same is valid for $\bar{f}$ ). These components appear to be connected by the relation:

$$
f\left(\beta_{1} \ldots \beta_{2 n+1}\right)_{n+1}^{1 \ldots 1} \underbrace{2 \ldots 2}_{n}=q^{n+1} f\left(\beta_{1} \ldots \beta_{2 n+1}\right)_{n+1}^{2 \ldots 2} \underbrace{2 \ldots 1}_{n}
$$

(the same for $\bar{f}$ ). The explicit formulas for $f_{n+1}^{1 \ldots 1} \underbrace{2 \ldots 2}_{n}$ and $\bar{f}_{n+1}^{1 \ldots 1} \underbrace{2 \ldots 2}_{n}$ are

$$
\begin{aligned}
& f\left(\beta_{1} \ldots \beta_{2 n+1}\right) \underbrace{1 \ldots 1}_{n+1} \underbrace{2 \ldots 2}_{n}=q^{\frac{n+1}{2}} c^{n} \prod_{i<j} \zeta\left(\beta_{i}-\beta_{j}\right) \\
& \quad \times \frac{1}{\prod_{i=1}^{n+1} \prod_{j=n+2}^{2 n+1} \operatorname{sh} \frac{\pi}{\xi}\left(\beta_{j}-\beta_{i}-\pi i\right)} \stackrel{f}{-\infty}^{\infty} d \alpha_{1} \ldots{\underset{-\infty}{f}}_{-\infty}^{\infty} d \alpha_{n} \prod_{i=1}^{n} \prod_{j=1}^{2 n+1} \varphi\left(\alpha_{i}-\beta_{j}\right) \\
& \quad \times \prod_{i<j} \operatorname{sh}\left(\alpha_{i}-\alpha_{j}\right) \exp \left\{-\left[\frac{1}{2}+\frac{\pi}{\xi}\left(n-\frac{3}{2}\right)\right] \sum_{i=1}^{n} \alpha_{i}\right. \\
&+ {\left.\left[\frac{1}{4}-\frac{\pi}{\xi}\left(n-\frac{3}{4}\right)\right] \sum_{j=1}^{n+1} \beta_{j}+\left[\frac{1}{4}-\frac{\pi}{\xi}\left(n-\frac{1}{4}\right)\right] \sum_{j=n+2}^{2 n+1} \beta_{j}\right\} } \\
& \quad P_{n}\left(\exp \left(\frac{2 \pi}{\xi} \alpha_{1}\right), \ldots \exp \left(\frac{2 \pi}{\xi} \alpha_{n}\right) \mid \exp \left(\frac{2 \pi}{\xi} \beta_{1}\right), \ldots\right. \\
&\left.\exp \left(\frac{2 \pi}{\xi} \beta_{n+1}\right) \mid \exp \left(\frac{2 \pi}{\xi} \beta_{n+2}\right), \ldots \exp \left(\frac{2 \pi}{\xi} \beta_{2 n+1}\right)\right)
\end{aligned}
$$




$$
\begin{aligned}
& \bar{f}\left(\beta_{1} \ldots \beta_{2 n+1}\right)_{n+1}^{1 \ldots 1} \underbrace{2 \ldots 2}_{n}=q^{\frac{n+1}{2}} c^{n} \prod_{i<j} \zeta\left(\beta_{i}-\beta_{j}\right) \\
& \times \frac{1}{\prod_{i=1}^{n+1} \prod_{j=n+2}^{2 n+1} \operatorname{sh} \frac{\pi}{\xi}\left(\beta_{j}-\beta_{i}-\pi i\right)} \overbrace{-\infty}^{\infty} d \alpha_{1} \ldots \stackrel{\wp}{-\infty}_{i=1}^{\infty} d \alpha_{n} \prod_{i=1}^{n} \prod_{j=1}^{2 n+1} \varphi\left(\alpha_{i}-\beta_{j}\right) \\
& \times \prod_{i<j} \operatorname{sh}\left(\alpha_{i}-\alpha_{j}\right) \exp \left\{-\left[\frac{\pi}{\xi}\left(n-\frac{1}{2}\right)-\frac{1}{2}\right] \sum_{i=1}^{n} \alpha_{i}\right. \\
& \left.+\left[-\frac{1}{4}-\frac{\pi}{\xi}\left(n+\frac{3}{4}\right)\right] \sum_{j=1}^{n+1} \beta_{j}+\left[-\frac{1}{4}-\frac{\pi}{\xi}\left(n-\frac{1}{4}\right)\right] \sum_{j=n+2}^{2 n+1} \beta_{j}\right\} \\
& \times Q_{n}\left(\exp \left(\frac{2 \pi}{\xi} \alpha_{1}\right), \ldots \exp \left(\frac{2 \pi}{\xi} \alpha_{n}\right) \mid \exp \left(\frac{2 \pi}{\xi} \beta_{1}\right), \ldots\right. \\
& \left.\exp \left(\frac{2 \pi}{\xi} \beta_{n+1}\right) \mid \exp \left(\frac{2 \pi}{\xi} \beta_{n+2}\right), \ldots \exp \left(\frac{2 \pi}{\xi} \beta_{2 n+1}\right)\right) \text {, }
\end{aligned}
$$

where

$$
\begin{aligned}
\zeta(\beta) & =\operatorname{sh} \frac{\beta}{2} \exp \left(\int_{0}^{\infty} \frac{\left(\sin ^{2} \frac{1}{2}(\beta+\pi i)+\operatorname{sh}^{2} \frac{\pi k}{4}\right) \operatorname{sh}\left(\frac{\pi-\xi}{2} k\right)}{k \operatorname{sh} \frac{\xi k}{2} \operatorname{sh} \pi k \operatorname{ch} \frac{\pi k}{2}} d k\right), \\
\varphi(\beta) & =\exp \left(-2 \int_{0}^{\infty} \frac{\left(\sin ^{2} \frac{1}{2} \beta k+\operatorname{sh}^{2} \frac{\pi k}{4}\right) \operatorname{sh}\left(\frac{\pi+\xi}{2} k\right)}{k \operatorname{sh} \frac{\xi k}{2} \operatorname{sh} \pi k} d k\right), \\
c & =\exp \left(-\int_{0}^{\infty} \frac{\operatorname{sh}^{2} \frac{\pi k}{4} \operatorname{sh}\left(\frac{\pi-\xi}{2} k\right)}{k \operatorname{sh} \frac{\xi k}{2} \operatorname{sh} \pi k \operatorname{ch} \frac{\pi k}{2}}\right)
\end{aligned}
$$

$f$ means special regularization of the integral [14];

$$
P_{n}\left(x_{1} \ldots x_{n}\left|a_{1} \ldots a_{n+1}\right| b_{1} \ldots b_{n}\right), Q_{n}\left(x_{1} \ldots x_{n}\left|a_{1} \ldots a_{n+1}\right| b_{1} \ldots b_{n}\right)
$$

are determinants of $n \times n$ matrices $\|A\|,\|B\|$ with the following matrix elements:

$$
\begin{gathered}
A_{i j}=A_{i}\left(x_{j}\left|a_{1} \ldots a_{n+1}\right| b_{1} \ldots b_{n}\right), \\
B_{i j}=B_{i}\left(x_{j}\left|a_{1} \ldots a_{n+1}\right| b_{1} \ldots b_{n}\right), \\
A_{i}\left(x\left|a_{1} \ldots a_{n+1}\right| b_{1} \ldots b_{n}\right)=\prod_{j=1}^{n+1}\left(x+q^{1 / 2} a_{j}\right) Q_{i-1}\left(x \mid b_{1} \ldots b_{n}\right) \\
+q^{i-1} \prod_{j=1}^{n}\left(x+q^{-1 / 2} b_{j}\right) Q_{i}\left(x \mid a_{1} \ldots a_{n+1}\right),
\end{gathered}
$$




$$
\begin{aligned}
& B_{i}\left(x\left|a_{1} \ldots a_{n+1}\right| b_{1} \ldots b_{n}\right)=\prod_{j=1}^{n+1}\left(x+q^{1 / 2} a_{j}\right) Q_{i}\left(x \mid b_{1} \ldots b_{n}\right) \\
& \quad+q^{i} \prod_{j=1}^{n}\left(x+q^{-1 / 2} b_{j}\right) Q_{i}\left(x \mid a_{1} \ldots a_{n+1}\right), \\
& Q_{i}\left(x \mid t_{1} \ldots t_{p}\right)=\sum_{k=0}^{i-1}\left(1-q^{(i-k)}\right) x^{i-k-1} q^{k / 2} \sigma_{k}\left(t_{1} \ldots t_{p}\right),
\end{aligned}
$$

$\sigma_{k}\left(t_{1} \ldots t_{p}\right)$ is the elementary symmetric polynomial of degree $k\left(\sigma_{k}\left(t_{1} \ldots t_{p}\right) \equiv 0\right.$ if $k<0$ or $k>p$ ).

To finish this section we would like to notice that in the limit $\xi \rightarrow \infty$ the group $S L(2)_{q}$ becomes the usual group $S L(2)$ SG becomes $S U(2)$ invariant Thirring model. The operators $\psi, \bar{\psi}$ in this limit give spin $\frac{1}{4}$ kinks.

\section{Parafermions in Scaling Three-State Potts Model}

Scaling three-state Potts model (SPM) is the perturbation of the CFT $M_{5}$ by the operator $\phi_{21}$ with scaling dimensions $\left(\frac{2}{5}, \frac{2}{5}\right)[21]$. This model has two particles in its spectrum: $\operatorname{particle}(p)$ and antiparticle $(\bar{p})$. The $p-p, p-\bar{p}, \bar{p}-\bar{p} S$-matrices are

$$
\begin{gathered}
S_{11}(\beta)=\frac{\operatorname{sh} \frac{1}{2}\left(\beta+\frac{2 \pi i}{3}\right)}{\operatorname{sh} \frac{1}{2}\left(\beta-\frac{2 \pi i}{3}\right)}, \quad S_{1-1}(\beta)=-\frac{\operatorname{sh} \frac{1}{2}\left(\beta+\frac{\pi i}{3}\right)}{\operatorname{sh} \frac{1}{2}\left(\beta-\frac{\pi i}{3}\right)}, \\
S_{-1-1}(\beta)=S_{11}(\beta) .
\end{gathered}
$$

The antiparticle is the bound state of two particles corresponding to the pole $\beta=\frac{2 \pi i}{3}$ in the $S$-matrix.

We consider this model because of two reasons: first, SPM is very interesting from a physical point of view; second, we want to show how can one generalize the construction described in the paper to models with bound states and different particles considering the simplest example.

The space of states is generated by the vectors

$$
\left|\beta_{n} \ldots \beta_{1}\right\rangle_{\varepsilon_{n} \ldots \varepsilon_{1}},
$$

where $\varepsilon_{i}= \pm 1$ distinguishes particle and antiparticle. The group $Z_{3}$ generated by the element $g\left(g^{3}=1\right)$ acts on the space of states as follows:

$$
T_{g}\left|\beta_{n} \ldots \beta_{1}\right\rangle=g^{\varepsilon_{n}} \ldots g^{\varepsilon_{1}}\left|\beta_{n} \ldots \beta_{1}\right\rangle_{\varepsilon_{n} \ldots \varepsilon_{1}} .
$$

Equations (2.4)-(2.6) on form factors of local operators can be easily generalized to this situation. But there is an additional equation which reflects the structure of bound states:

$$
\begin{aligned}
& \text { res } \\
\beta_{n}=\beta_{n-1}+\frac{2 \pi i}{3} & f\left(\beta_{1} \ldots \beta_{n-1}, \beta_{n}\right)_{\varepsilon_{1} \ldots \varepsilon_{n-1} \varepsilon_{n}} \\
= & 2^{-1} 3^{1 / 4} \pi^{-1 / 2} \delta_{\varepsilon_{n} \varepsilon_{n-1}} f\left(\beta_{1} \ldots \beta_{n-2}, \beta_{n-1}+\frac{\pi i}{3}\right)_{\varepsilon_{1} \ldots \varepsilon_{n-2},-\varepsilon_{n-1}} .
\end{aligned}
$$


The form factors of physically important operators for SPM were calculated in the preprint [25]. The form factors of $T_{\mu \nu}$ can be expressed in terms of the function $g$ (2.10) which is given by

$$
\begin{aligned}
g\left(\beta_{1} \ldots \beta_{n}\right)_{\varepsilon_{1} \ldots \varepsilon_{n}}=c^{n} \prod_{i<j} \zeta_{\varepsilon_{i} \varepsilon_{j}}\left(\beta_{i}-\beta_{j}\right) \\
\quad \times P\left(\left\{\exp \left(\beta_{j}\right)\right\}_{j: \varepsilon_{j}=1} \mid\left\{\exp \left(\beta_{j}\right)\right\}_{j: \varepsilon_{j}=-1}\right) \\
\quad \times \exp \left(-\left(\frac{1}{6}(2 k+\ell-6) \sum_{j: \varepsilon_{j}=1} \beta_{j}+\frac{1}{6}(2 \ell+k-6) \sum_{j: \varepsilon_{j}=-1} \beta_{j}\right)\right),
\end{aligned}
$$

where $k(\ell)$ is the number of particles (antiparticles),

$$
\begin{gathered}
\zeta_{11}(\beta)=\zeta_{-1-1}(\beta)=\frac{\operatorname{sh} \frac{1}{2} \beta}{2 \operatorname{sh} \frac{1}{2}\left(\beta-\frac{2 \pi i}{3}\right) \operatorname{sh} \frac{1}{2}\left(\beta+\frac{2 \pi i}{3}\right)} \\
\times \exp \left(\frac{1}{2} \int_{0}^{\infty} \frac{\left(\sin ^{2} \frac{1}{2}(\beta+\pi i) k+\frac{2}{3} \operatorname{sh}^{2} \frac{\pi k}{3}\right) \operatorname{sh} \frac{\pi k}{3}}{k(\operatorname{sh} \pi k)^{2}} d k\right), \\
\zeta_{1-1}(\beta)=\zeta_{-11}(\beta)=\operatorname{ch} \frac{\beta}{2} \zeta_{11}\left(\beta-\frac{\pi i}{3}\right) \zeta_{11}\left(\beta+\frac{\pi i}{3}\right), \\
c=\left(\frac{1}{2 \pi}\right)^{1 / 3} \exp \left(-\frac{1}{3} \int \frac{\operatorname{sh}^{3} \frac{\pi k}{3}}{k(\operatorname{sh} \pi k)^{2}} d k\right) .
\end{gathered}
$$

$Z_{3}$ symmetry of $T_{\mu \nu}$ require that $\sum \varepsilon_{j} \equiv 0(\bmod 3)$. The function $P\left(a_{1} \ldots a_{k} \mid b_{1} \ldots b_{\ell}\right)$ is equal to $\left(a_{1}+b_{1}\right)^{-1}$ for $k=\ell=1$, for $k+\ell>2$ it is given by the integral

$$
\begin{gathered}
P\left(a_{1} \ldots a_{k} \mid b_{1} \ldots b_{\ell}\right)=\left(\frac{1}{2 \pi i}\right)^{k+\ell-4} \int_{c} d t_{1} \ldots \int_{c} d t_{\frac{2 k+\ell}{3}-2} \\
\int_{c} d u_{1} \ldots \int_{c} d u_{2 \ell+k}-2 \prod_{i} t_{i}^{-3 i} \prod_{i} u_{i}^{-3 i} \prod_{i, j}\left(t_{i}-a_{j}\right) \\
\times \prod_{i, j}\left(u_{i}+b_{j}\right) \prod_{i<j}\left(u_{i}-u_{j}\right) \prod_{i<j}\left(t_{i}-t_{j}\right) \prod_{i, j}\left(t_{i}-u_{j}\right),
\end{gathered}
$$

where the contour $c$ surrounds zero, i.e. all the integrals are equal to the residues at the points $t_{i}=0, u_{i}=0$. Actually, $P\left(a_{1} \ldots a_{k} \mid b_{1} \ldots b_{\ell}\right)$ is a polynomial and the integral (5.2) presents the polynomial in a compact form. Another useful form of $P\left(a_{1} \ldots a_{3 m}\right)(\ell=0)$ is given in [25]: $P$ is equal to the determinant of $m \times m$ matrix $\|M\|$ with the following matrix elements:

$$
M_{i j}=\sigma_{3 p-q-\left[\frac{q-1}{2}\right]}\left(x_{1} \ldots x_{3 m}\right),
$$

$\sigma_{k}$ is an elementary symmetric polynomial of degree $k$. 
Now let us construct parafermions. We have two particles, that is why we shall construct two parafermionic operators $\psi_{\varepsilon}$ :

$$
f_{\varepsilon}\left(\beta_{1} \ldots \beta_{n-1}\right)_{\varepsilon_{1} \ldots \varepsilon_{n-1}}=\lim _{\beta_{n} \rightarrow \infty} e^{\Delta \beta_{n}} g\left(\beta_{1} \ldots \beta_{n-1}, \beta_{n}\right)_{\varepsilon_{1} \ldots \varepsilon_{n-1}, \varepsilon}
$$

explicit calculation using (5.2) shows that $\Delta=\frac{2}{3}$. Similarly we have two operators $\bar{\psi}_{\varepsilon}$ with form factors

$$
\bar{f}_{\varepsilon}\left(\beta_{1} \ldots \beta_{n-1}\right)_{\varepsilon_{1} \ldots \varepsilon_{n-1}}=\lim _{\beta_{n} \rightarrow-\infty} e^{-\Delta \beta_{n}} g\left(\beta_{1} \ldots \beta_{n-1}, \beta_{n}\right)_{\varepsilon_{1} \ldots \varepsilon_{n-1}, \varepsilon} .
$$

Returning to the formal procedure of Sect. 3 we realize that now we have two representations $\varrho_{+}: \varrho_{+}^{\varepsilon}$ associated with two particles

$$
\varrho_{+}^{\varepsilon}\left(g^{\varepsilon^{\prime}}\right)=\lim _{\beta \rightarrow \infty} S_{\varepsilon \varepsilon^{\prime}}(\beta)=\exp \left(\frac{2 \pi i}{3} \varepsilon \varepsilon^{\prime}\right) .
$$

Similarly there are two representations $\varrho_{-}: \varrho_{-}^{\varepsilon}$ :

$$
\varrho_{-}^{\varepsilon}\left(g^{\varepsilon^{\prime}}\right)=\lim _{\beta \rightarrow-\infty} S_{\varepsilon \varepsilon^{\prime}}(\beta)=\exp \left(-\frac{2 \pi i}{3} \varepsilon \varepsilon^{\prime}\right) .
$$

The operators $\psi_{\varepsilon}, \bar{\psi}_{\varepsilon}$ satisfy the following equal time commutation relations:

$$
\begin{aligned}
& \psi_{\varepsilon}(x) \psi_{\varepsilon^{\prime}}(y)=\exp \left(\frac{2 \pi i}{3} \varepsilon \varepsilon^{\prime}\right) \psi_{\varepsilon^{\prime}}(y) \psi_{\varepsilon}(x), x<y ; \\
& \bar{\psi}_{\varepsilon}(x) \bar{\psi}_{\varepsilon^{\prime}}(y)=\exp \left(\frac{2 \pi i}{3} \varepsilon \varepsilon^{\prime}\right) \bar{\psi}_{\varepsilon^{\prime}}(y) \bar{\psi}_{\varepsilon}(x), x>y ; \\
& \psi_{\varepsilon}(x) \bar{\psi}_{\varepsilon^{\prime}}(y)=\exp \left(\frac{2 \pi i}{3} \varepsilon \varepsilon^{\prime}\right) \bar{\psi}_{\varepsilon^{\prime}}(y) \psi_{\varepsilon}(x), \forall x, y .
\end{aligned}
$$

These operators have spins $\left(\frac{2}{3}, 0\right),\left(0, \frac{2}{3}\right)$ and give in the scaling limit the parafermionic currents considered in [17].

From the formula (5.1) one easily gets the formulas for the form factors of $\psi_{\varepsilon}, \bar{\psi}_{\varepsilon}$.

$$
\begin{aligned}
& f_{1}\left(\beta_{1} \ldots \beta_{n}\right)_{\varepsilon_{1} \ldots \varepsilon_{n}}=c^{n} \prod_{i<j} \zeta_{\varepsilon_{i} \varepsilon_{j}}\left(\beta_{i}-\beta_{j}\right) \\
& \quad \times P_{1}\left(\left\{\exp \left(\beta_{j}\right)\right\}_{j: \varepsilon_{j}=1} \mid\left\{\exp \left(\beta_{j}\right)\right\}_{j: \varepsilon_{j}=-1}\right) \\
& \quad \times \exp \left(-\left(\frac{1}{6}(2 k+\ell-2) \sum_{j: \varepsilon_{j}=1} \beta_{j}+\frac{1}{6}(2 \ell+k-4) \sum_{j: \varepsilon_{j}=-1} \beta_{j}\right)\right) \\
& \bar{f}_{1}\left(\beta_{1} \ldots \beta_{n}\right)_{\varepsilon_{1} \ldots \varepsilon_{n}}=c^{n} \prod_{i<j} \zeta_{\varepsilon_{i} \varepsilon_{j}}\left(\beta_{i}-\beta_{j}\right) \\
& \times \bar{P}_{1}\left(\left\{\exp \left(\beta_{j}\right)\right\}_{j: \varepsilon_{j}=1} \mid\left\{\exp \left(\beta_{j}\right)\right\}_{j: \varepsilon_{j}=-1}\right) \\
& \times \exp \left(-\left(\frac{1}{6}(2 k+\ell-6) \sum_{j: \varepsilon_{j}=1} \beta_{j}+\frac{1}{6}(2 \ell+k-6) \sum_{j: \varepsilon_{j}=-1} \beta_{j}\right)\right), \\
& \quad f_{-1}\left(\beta_{1} \ldots \beta_{n}\right)_{\varepsilon_{1} \ldots \varepsilon_{n}}=f_{1}\left(\beta_{1} \ldots \beta_{n}\right)_{-\varepsilon_{1} \ldots-\varepsilon_{n}}, \\
& \quad \bar{f}_{-1}\left(\beta_{1} \ldots \beta_{n}\right)_{\varepsilon_{1} \ldots \varepsilon_{n}}=\bar{f}_{1}\left(\beta_{1} \ldots \beta_{n}\right)_{-\varepsilon_{1} \ldots-\varepsilon_{n}},
\end{aligned}
$$


where

$$
\begin{aligned}
& P_{1}\left(a_{1} \ldots a_{k} \mid b_{1} \ldots b_{\ell}\right)=\left(\frac{1}{2 \pi i}\right)^{k+\ell-3} \int_{c} d t_{1} \ldots \int_{c} d \frac{t 2 k+\ell-4}{3} \\
& \int_{c} d u_{1} \ldots \int_{c} d u \frac{2 \ell+k-5}{3} \prod_{i} t_{i}^{-3 i} \prod u_{i}^{-3 i} \prod_{i, j}\left(t_{i}-a_{j}\right) \\
& \times \prod\left(u_{i}+b_{j}\right) \prod_{i<j}\left(u_{i}-u_{j}\right) \prod_{i<j}\left(t_{i}-t_{j}\right) \prod_{i, j}\left(t_{i}-u_{j}\right), \\
& \bar{P}_{1}\left(a_{1} \ldots a_{k} \mid b_{1} \ldots b_{\ell}\right)=\left(\frac{1}{2 \pi i}\right)^{k+\ell-3} \int_{c} d t_{1} \ldots \int_{c} d t \frac{2 k+\ell-4}{3} \\
& \int_{c} d u_{1} \ldots \int_{c} d \frac{2 l+k-5}{3} \prod_{i} t_{i}^{-3 i+1} \prod_{i} u_{i}^{-3 i} \prod_{i, j}\left(t_{i}-a_{j}\right) \\
& \times \prod_{i, j}\left(u_{i}+b_{j}\right) \prod_{i<j}\left(u_{i}-u_{j}\right) \prod_{i<j}\left(t_{i}-t_{j}\right) \prod_{i, j}\left(t_{i}-u_{j}\right) .
\end{aligned}
$$

The last two lines in (5.3) follow from the equations

$$
\psi_{\varepsilon}(x)=C \psi_{-\varepsilon}(x) C, \bar{\psi}_{\varepsilon}(x)=C \bar{\psi}_{-\varepsilon}(x) C,
$$

where $C$ is the operator of charge conjugation which changes particles to antiparticles.

\section{Additional Remarks}

We have shown that the generalized statistics of physical particles leads to the generalized statistics of local operators and have presented the explicit construction of these operators. Let us return to the construction. What we have really done is test the energy-momentum tensor by the particle with large momentum (which is becoming light-like). Let us denote the result of the testing by $\langle T\rangle_{\beta}(\beta \rightarrow \pm \infty)$. We have shown that for the models considered

$$
\left\langle T_{++}(0)\right\rangle_{\beta} \underset{\beta \rightarrow \infty}{\sim} e^{(2-\Delta) \beta} \psi(0) .
$$

It is interesting to study the result of the testing for other models. Here several possibilities arise.

For a pure bosonic model (for example sh-Gordon model [26]) one gets using the formulas for form factors,

$$
\left\langle T_{++}(0)\right\rangle_{\beta} \underset{\beta \rightarrow \infty}{\sim} e^{\beta} \partial_{+} \varphi(0)
$$

where $\varphi$ is the Bose field.

There are models with bosonic spectra containing the interaction of $\varphi^{3}$ type (for example RSG $(2 / 2 n+1)$ [18], $E_{8}$-model [27]). For these models we have

$$
\left\langle T_{++}(0)\right\rangle_{\beta} \underset{\beta \rightarrow \infty}{\sim} \text { const } e^{2 \beta}+e^{\beta} A(0),
$$

but the operator $A$ appears to be nonlocal (its two-particle form factor has a pole). 
Actually, the operator $A$ can be expressed in terms of $T_{++}$:

$$
A(\xi, \eta)=\int_{-\infty}^{\xi} T_{++}\left(\xi^{\prime}, \eta\right) d \xi^{\prime} .
$$

Certainly it is very natural that we cannot obtain a local operator by means of (6.1) for this kind of model. Otherwise we have an operator which gives spin 1 current in the scaling limit and the theory cannot be a perturbation of minimal models of CFT which is supposed about RSG $(2 / 2 n+1), E_{8}$ model. Generally it is clear from these reasonings that the perturbations of the minimal models of CFT should either have generalized statistics physical particles or contain the interaction of $\varphi^{3}$-type.

Finally, there are models which contain logarithms in the leading terms of ultraviolet asymptotics. For example for the $O(3)$ nonlinear $\sigma$-model one gets [16]:

$$
\left\langle T_{++}\right\rangle_{\beta} \underset{\beta \rightarrow \infty}{\sim} \frac{e^{2 \beta}}{\beta} n
$$

where $n$ is an $n$-field.

Another point is that the construction presented in the paper can be used for getting parafermionic fields with higher spins. For example in SG model one can consider the limit of the energy momentum tensor form factors when the rapidities of $(2 j+1)$ particles become much greater than the rest of rapidities and project onto $S L(2)_{q}$ a spin $j$ representation with respect to these particles. Then one gets a spin $j$ [with respect to $S L(2)_{q}$ ] parafermion whose Lorenz spin can be calculated and appears to be equal to $2 j \frac{(2 j+1) \pi+(2 j-1) \xi}{\xi}$. For $\xi=\frac{\pi r}{r_{1}}$ these dimensions coincide with the dimensions $\Delta_{2 j+1,1}$ in $M_{r, r+r_{1}}$.

It is possible also to consider the limit when the rapidities of $\left(2 j_{1}+1\right)$ particles go to plus infinity while the rapidities of $\left(2 j_{2}+1\right)$ particles go to minus infinity and project onto $S L(2)_{q}$ spin $j_{1}$ and spin $j_{2}$ representations respectively. Then one gets the operator $O_{m_{1} m_{2}}^{j_{1} j_{2}}(x)$ which transforms under the tensor product of spin $j_{1}$ and spin $j_{2}$ representations. This is the most general situation (parafermions correspond to $j_{1}$ or $j_{2}$ equal to zero). Unfortunately it is difficult to calculate the scaling dimensions of these operators for general $j_{1}, j_{2}$ : they are not parafermionic ones and the investigation of short distance behaviour of Green functions becomes a complicated problem. It is possible, however, to generalize the reasonings of Sect. 3 and to calculate the equal time commutative relations of these operators:

$$
\begin{aligned}
& \sum_{n_{2}, n_{1}^{\prime}, n_{2}^{\prime}, \ell} O_{m_{1} n_{2}}^{j_{1} j_{2}}(x) O_{n_{1}^{\prime} n_{2}^{\prime}}^{j_{1}^{\prime} j_{2}^{\prime}}(y)\left(R^{j_{2} j_{1}^{\prime}}\right)_{\ell m_{1}^{\prime}}^{n_{2} n_{1}}\left(R^{j_{2} j_{2}^{\prime}}\right)_{m_{2} m_{2}^{\prime}}^{\ell n_{2}^{\prime}} \\
& \quad=\sum_{n_{1}, n_{1}^{\prime}, n_{2}^{\prime}, \ell} O_{n_{1}^{\prime} n_{2}^{\prime}}^{j_{1}^{\prime} j_{2}^{\prime}}(x) O_{n_{1} m_{2}}^{j_{1} j_{2}}(y)\left(R^{j_{1} j_{1}^{\prime}}\right)_{m_{1} m_{1}^{\prime}}^{\ell n_{1}^{\prime}}\left(R^{j_{1} j_{2}^{\prime}}\right)_{\ell m_{2}^{\prime}}^{n_{1} n_{2}^{\prime}}, \\
& x<y .
\end{aligned}
$$

The relations (0.4) are particular cases of the relation (6.2). The operators $O_{m_{1} m_{2}}^{j_{1} j_{2}}$ generate local spinless operators

$$
O^{j}(x)=\sum_{m=-j / 2}^{j / 2} q^{m} O_{m-m}^{j j}(x) .
$$


These operators for RSG model should give spinless operators $\phi_{2 j+1,1}$ in the scaling limit.

Finally, let us notice that every invariant under the action of the quantum group operator generates the associated operators via the same procedure. In particular the disorder operator in SPM being $Z_{3}$ invariant generates the order operator. The operators $\exp \left(\frac{i k \sqrt{\gamma} \varphi}{2}\right), k \geqq 1$ in SG model which give for the reduced model the operators having $\phi_{1, k+1}$ as scaling limits generate the operators giving in the scaling limit $\phi_{2 j+1, k+1}$.

Acknowledgements. The author is grateful to L. D. Faddeev, A. N. Kirillov, N. Yu. Reshetikhin, L. A. Takhtajan for helpful discussions.

\section{References}

1. Swieca, A.: Fortschr. Phys. 25, 303 (1977)

2. Karowski, M., Thur, H.J.: Nucl. Phys. B 109, 61 (1981)

3. Köberll, R., Kurak, V., Swieca, A.: Phys. Rev. D 20, 897 (1979)

4. Tsuchiya, A., Kanie, Y.: Adv. Stud. Pure Math. 16, 297 (1988)

5. Rehren, K., Schroer, B.: Berlin Preprint FU-88-0439 (1988)

6. Moore, G., Reshetikhin, N.: Princeton Preprint IASSNS-HEP-89/18 (1989)

7. Zamolodchikov, A.B., Zamolodchikov, Al.B.: Ann. Phys. 120, 253 (1979)

8. Yang, C.N.: Phys. Rev. 168, 1920 (1968)

9. Baxter, R.J.: Exactly solved models in statistical mechanics. London: Academic Press 1982

10. Drinfeld, V.G.: Doklady Academii Nauk SSSR 283, 1060 (1985)

11. Jimbo, M.: Lett. Math. Phys. 10, 63 (1985)

12. Faddeev, L.D., Reshitikhin, N.Yu., Takhtajan, L.A.: Algebra Analysis 1, 178 (1989)

13. Reshetikhin, N.Yu., Smirnov, F.A.: Commun. Math. Phys. (to appear)

14. Smirnov, F.A.: J. Phys. A 19, L575 (1986); Zap. Nauch. Sem. LOMI 165, 98 (1987)

15. Kirillov, A.N., Smirnov, F.A.: Phys. Lett. 198 B, 506 (1987); Zap. Nauch. Semin. LOMI 164, 80 (1987)

16. Kirillov, A.N., Smirnov, F.A.: Int. J. Mod. Phys. A 3, 731 (1988)

17. Fateev, V.A., Zamolodchikov, A.B.: ZhETF 89, 380 (1985); ZhETF 90, 1553 (1986)

18. Smirnov, F.A.: Preprint LOMI E-4-89 (1989)

19. Zamolodchikov, A.B.: Pisma JhETF 43, 565 (1986)

20. Zamolodchikov, A.B.: Pisma JhETF 46, 129 (1987)

21. Zamolodchikov, A.B.: Int. J. Mod. Phys. A 3, 720 (1988)

22. Faddeev, L.D., Sklyanin, E.K., Takhtajan, L.A.: Teor. Mat. Fiz. 40, 194 (1979)

23. Kirillov, A.N., Reshetikhin, N.Yu.: Preprint LOMI E-9-88 (1988)

24. Andrews, C., Baxter, R., Forrester, D.: J. Stat. Phys. 35, 193 (1984)

25. Kirillov, A.N., Smirnov, F.A.: ITF preprint ITF-88-73R, Kiev (1988)

26. Khamitov, I.M.: Zap. Nauch. Semin. LOMI 146, 147 (1985)

Kirillov, A.N.: Zap. Nauch. Semin. LOMI 146, 9 (1985)

27. Zamolodchikov, A.B.: Rutherford Appleton Laboratory Preprint RAL-89-001 (1989)

Communicated by N. Yu. Reshetikhin 
